\title{
Broadband quasi-phase-matching in dispersion-engineered all-optically poled silicon nitride waveguides
}

\author{
Edgars Nitiss, ${ }^{1}$ Boris Zabelich, ${ }^{1}$ Ozan Yakar, ${ }^{1}$ Junqiu Liu, ${ }^{2}$ Rui Ning Wang, ${ }^{2}$ Tobias J. Kippenberg, ${ }^{2}$ \\ and Camille-Sophie Brès ${ }^{1, *}$
}

${ }^{1}$ Ecole Polytechnique Fédérale de Lausanne (EPFL), Photonic Systems Laboratory (PHOSL), STI-IEL, Station 11, CH-1015 Lausanne, Switzerland ${ }^{2}$ Ecole Polytechnique Fédérale de Lausanne (EPFL), Laboratory of Photonics and Quantum Measurements (LPQM), SB-IPHYS,

Station 3, CH-1015 Lausanne, Switzerland

${ }^{*}$ Corresponding author: camille.bres@epfl.ch

Received 29 April 2020; revised 11 June 2020; accepted 10 July 2020; posted 10 July 2020 (Doc. ID 396489); published 20 August 2020

\begin{abstract}
Quasi-phase-matching (QPM) has become one of the most common approaches for increasing the efficiency of nonlinear three-wave mixing processes in integrated photonic circuits. Here, we provide a study of dispersion engineering of QPM second-harmonic $(\mathrm{SH})$ generation in stoichiometric silicon nitride $\left(\mathrm{Si}_{3} \mathrm{~N}_{4}\right)$ waveguides. We apply waveguide design and lithographic control in combination with the all-optical poling technique to study the QPM properties and shape the waveguide dispersion for broadband spectral conversion efficiency inside $\mathrm{Si}_{3} \mathrm{~N}_{4}$ waveguides. By meeting the requirements for maximal bandwidth of the conversion efficiency spectrum, we demonstrate that group-velocity matching of the pump and SH is simultaneously satisfied, resulting in efficient SH generation from ultrashort optical pulses. The latter is employed for retrieving a carrier-envelope-offset frequency of a frequency comb by using an $f-2 f$ interferometric technique, where supercontinuum and SH of a femtosecond pulse are generated in $\mathrm{Si}_{3} \mathrm{~N}_{4}$ waveguides. Finally, we show that the waveguide dispersion determines the QPM wavelength variation magnitude and sign due to the thermo-optic effect. () 2020 Chinese Laser Press
\end{abstract}

https://doi.org/10.1364/PRJ.396489

\section{INTRODUCTION}

In recent years, photonic integrated circuits have experienced significant advancements, driven by the promise of compactness, efficiency, low cost, and mass production. A wide range of passive and active functionalities, from electro-optical modulation to frequency conversion, has been demonstrated on various platforms such as silicon [1,2], stoichiometric silicon nitride $\left(\mathrm{Si}_{3} \mathrm{~N}_{4}\right)[3,4]$, lithium niobate $(\mathrm{LN})[5,6]$, and aluminum nitride [7-9]. Many of these functionalities are already available within a standard full-wafer technology at foundries. The control of nonlinear effects in these integrated platforms, either based on the second- or third-order susceptibilities $\left(\chi^{(2)}\right.$ and $\chi^{(3)}$, respectively), represents a large area of research, as frequency mixing effects offer a practical solution for light generation. In an effort to access three-wave mixing effects, for communication or quantum applications, the abovementioned materials are being studied for the demonstration of an efficient and compact $\chi^{(2)}$ waveguide. The highest conversion efficiencies (CEs) are typically achieved in devices that employ quasi-phase-matching (QPM) $[5,10]$, which is a technique where, by creating a periodic structure in a nonlinear medium, the phase mismatch between frequencies participating in the targeted three-wave mixing process is compensated, allowing for efficient energy transfer [11]. The efficiency of nonlinear interaction in waveguide devices can be characterized by second-harmonic $(\mathrm{SH})$ generation. Despite the demonstration of CE reaching thousands of \%/W, there still have been a limited number of reports on the properties of QPM such as CE bandwidth for continuous wave $(\mathrm{CW})$ or short pulse regimes, along with thermo-optic tuning of QPM. One of the reasons for the limited number of studies is that the waveguide devices typically require complex nanostructuring techniques in order to ensure periodic modulation of nonlinearity for QPM. Recently, we demonstrated that efficient QPM SH generation in $\mathrm{Si}_{3} \mathrm{~N}_{4}$ waveguides can be achieved by using all-optical poling $[12,13]$, which was also confirmed by other groups [14-16]. Here, an effective nonlinear $\chi^{(2)}$ grating builds up spontaneously in the presence of a high-intensity coherent pump and weak SH due to the coherent photogalvanic effect [17-19]. The method is simple to implement and allows inscription of self-organized nonlinear gratings for QPM at a selected pump wavelength in a manner of minutes [20]. This method is therefore useful for studying the influence of dispersion on QPM properties, without the need for fabricating many samples. 
The method does require the waveguide to be able to withstand high optical intensities with peak power reaching the level of tens of watts, as is the case for the $\mathrm{Si}_{3} \mathrm{~N}_{4}$ waveguides used in our study. The additional strong points of the platform, such as excellent lithographic control, low propagation loss, and high third-order nonlinearity, have indeed established $\mathrm{Si}_{3} \mathrm{~N}_{4}$ as a perfect candidate for various applications, including low pulse energy supercontinuum (SC) generation [21-24], frequency comb generation [3,25], ultralow-loss resonators [26-29], and entangled photon-pair generation [30,31].

Here, we combine the strengths of all-optical poling and those of the $\mathrm{Si}_{3} \mathrm{~N}_{4}$ waveguide platform in order to study the QPM properties depending on waveguide dispersion, carefully controlled through altering the waveguide dimensions. Until now, the concept of optimizing QPM by dispersion engineering in integrated photonic waveguides has rarely been employed and most exclusively for group-velocity (GV) matching of short pulses [16,32]. In this work, we show, numerically and experimentally, that the bandwidth of the CE spectrum in a QPM structure can be controlled and significantly enhanced solely by geometric dispersion engineering. By meeting the requirements for maximal $\mathrm{CE}$ bandwidth for $\mathrm{CW}$ operation, we demonstrate that GV matching for a short-pulsed pump and $\mathrm{SH}$ is simultaneously satisfied. The latter provides means for $\mathrm{SH}$ generation from a $\mathrm{CW}$ signal, i.e., long and ultrashort optical pulses, without sacrificing efficiency of either regime. As a proof-of-concept, we employ this phenomenon to retrieve carrier-envelope-offset frequency $\left(f_{\mathrm{CEO}}\right)$ of a frequency comb by using an $f-2 f$ interferometric technique, where SC and $\mathrm{SH}$ of a femtosecond pulse are generated in $\mathrm{Si}_{3} \mathrm{~N}_{4}$ waveguides. Finally, we address the thermo-optic tuning properties of QPM that are also shown to vary significantly with the waveguide dispersion.

\section{QPM SPECTRAL RESPONSE}

\section{A. Theoretical Considerations}

The CE of a nearly monochromatic optical pump in a QPM device is calculated by [20]

$$
\mathrm{CE}=\frac{\left(\omega L_{g} \chi_{\mathrm{eff}}^{(2)}\right)^{2}}{2 \varepsilon_{o} c^{3} n_{\omega}^{2} n_{2 \omega}} \frac{A_{2 \omega}}{A_{\omega}^{2}}\left[\operatorname{sinc}\left(\frac{\Delta \beta L_{g}}{2}\right)\right]^{2},
$$

where $\omega$ is the pump frequency, $L_{g}$ is the length of the QPM structure, $\chi_{\text {eff }}^{(2)}$ is the effective nonlinearity, $n_{\omega}$ and $n_{2 \omega}$ are the effective refractive indices at pump and $\mathrm{SH}$ wavelengths, respectively, $A_{\omega}$ and $A_{2 \omega}$ are the effective mode areas at pump and $\mathrm{SH}$ wavelengths [5], respectively, and $\Delta \beta$ is the propagation constant mismatch. In Eq. (1), $\Delta \beta$ is expressed as

$$
\Delta \beta=\beta_{2 \omega}-2 \beta_{\omega}-\frac{2 \pi}{\Lambda},
$$

where $\Lambda$ is the grating period, and $\beta_{2 \omega}$ and $\beta_{\omega}$ are the propagation constants at the $\mathrm{SH}$ and fundamental harmonic, respectively [11]. After all-optical poling, by definition $\Delta \beta=0$ at the QPM wavelength, which is identical to poling wavelength in case the QPM measurement and poling are done at the same sample temperature. As evident from Eq. (1), the amplitude of CE efficiency is determined by the effective nonlinearity $\chi_{\text {eff }}^{(2)}$ as well as the grating length $L_{g}$, while the CE bandwidth can be calculated by sinc-squared function and is determined by $L_{g}$ and the propagation constant mismatch $\Delta \beta$ around the QPM wavelength. A longer grating length will result in higher efficiency but will also have a reduced conversion bandwidth. Evidently, the $\Delta \beta$ variation near the QPM wavelength has a crucial influence on the conversion bandwidth. In order to maintain a large bandwidth in such a QPM structure without sacrificing efficiency, the change of $\Delta \beta$ relative to the wavelength [i.e., $\partial(\Delta \beta) / \partial \lambda$ ] should be equal to zero. This is met when the following equation is satisfied [11]:

$$
\frac{n_{2 \omega}-n_{\omega}}{\lambda}+\frac{\partial n_{\omega}}{\partial \lambda}-\frac{1}{2} \frac{\partial n_{2 \omega}}{\partial \lambda}=0,
$$

where $\lambda$ is the QPM wavelength. We will further refer to the wavelength at which Eq. (3) is satisfied as $\lambda_{0}$.

The $\Delta \beta$ dependence on wavelength is determined not only by the refractive index of the employed material but also by the waveguide geometry. In Fig. 1, we plot the expected variation of the QPM response for all-optically poled $\mathrm{Si}_{3} \mathrm{~N}_{4}$ waveguides. In order to match the subsequent experiments, we ran simulations in the telecommunication band. Here, all the simulations for different waveguide geometry were done using a numerical mode solver. We first consider poling the waveguide at $1.55 \mu \mathrm{m}$ : after poling, $\Delta \beta=0$ at this particular wavelength and at the employed light polarization, transverseelectric (TE) or transverse-magnetic (TM), following the alloptical inscription of the self-organized grating with period $\Lambda=\lambda /\left[2 \cdot\left(n_{2 \omega}-n_{\omega}\right)\right]$. In Figs. 1(a) and 1(d), we map the $\Delta \beta$ dependence on waveguide width and wavelength, for poling performed on TE or TM polarization, respectively. The height of the waveguide is fixed at $0.75 \mu \mathrm{m}$, grating length fixed at $L_{g}=10 \mathrm{~mm}$, and effective nonlinearity at $\chi_{\text {eff }}^{(2)}=0.1 \mathrm{pm} / \mathrm{V}$. The resulting CE according to Eq. (1) is shown in Figs. 1(b) and 1(e) corresponding to TE and TM cases, respectively. As expected, the maximum $\mathrm{CE}$ decreases as the cross-section of the waveguide is increased. This occurs because the modes become less confined in larger waveguides causing the light intensity inside the core to drop. The CE maps in Figs. 1(b) and 1(e) show that variation of waveguide geometry can have significant influence on the CE bandwidth. As evident, a broad QPM spectral response can be achieved for TE polarized light in a waveguide with cross-section of around $1.6 \mu \mathrm{m} \times 0.75 \mu \mathrm{m}$. In contrast, the QPM spectral response for TM polarized light in a waveguide with the same dimensions is significantly narrower. In Figs. 1(c) and 1(f), the CE in waveguides with height $0.75 \mu \mathrm{m}$ and selected widths of 1.6, 1.8, and $2.0 \mu \mathrm{m}$ is shown for TE and TM polarized light, respectively. By comparing the CE spectral response when operated with TE light in waveguides with these dimensions, we see that the $\mathrm{CE}$ spectral response narrows significantly as the waveguide width is increased from 1.6 to $2.0 \mu \mathrm{m}$. The opposite takes place when QPM is induced for the TM case, i.e., the CE spectral bandwidth experiences slight increase in width but remains relatively narrow.

The CE spectral bandwidth and shape could also vary considerably for a given waveguide depending on the QPM wavelength, with more dramatic changes when operating near $\lambda_{0}$. In Fig. 2, we show the CE dependence on wavelength in waveguides 
(a)

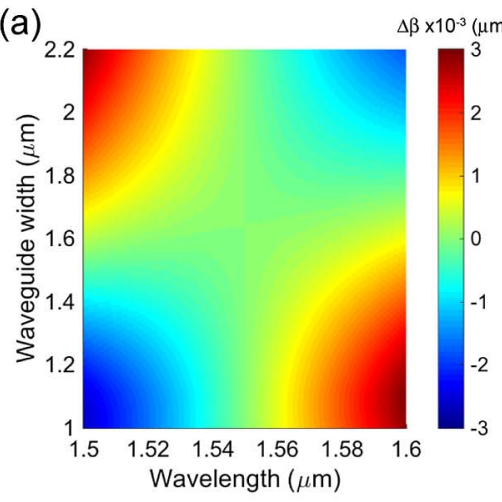

(d)

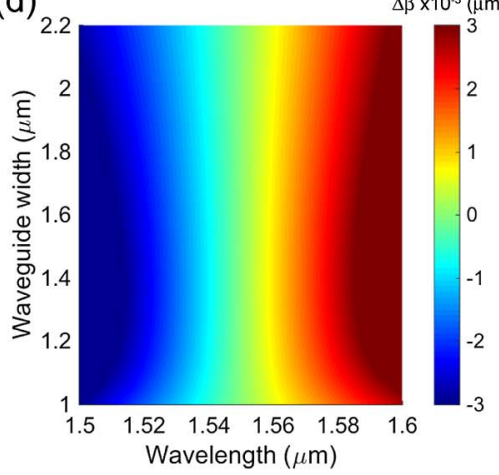

(b)

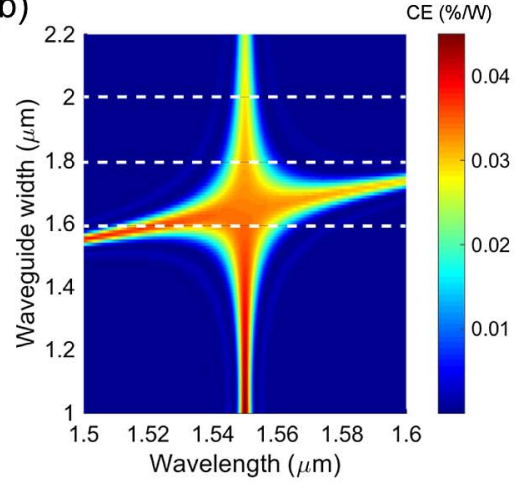

(e)

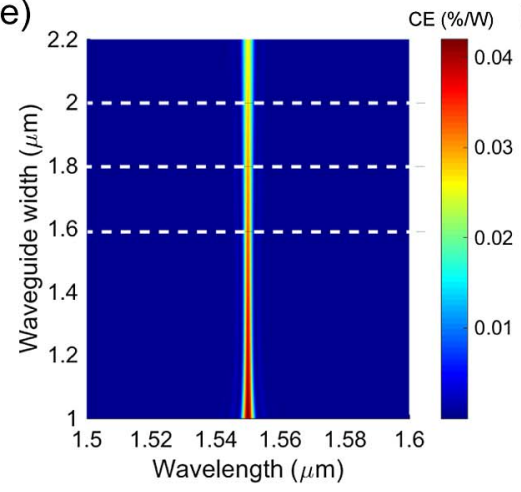

(c)

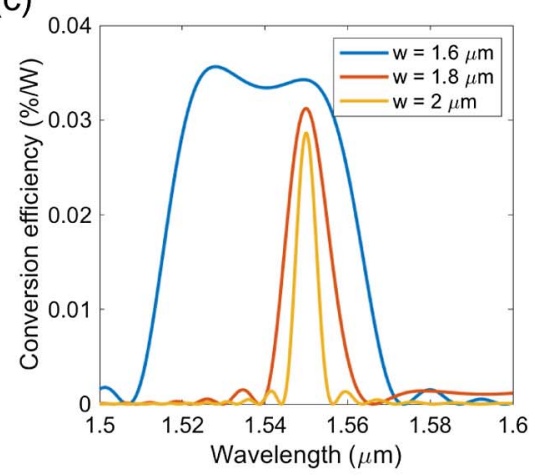

(f)

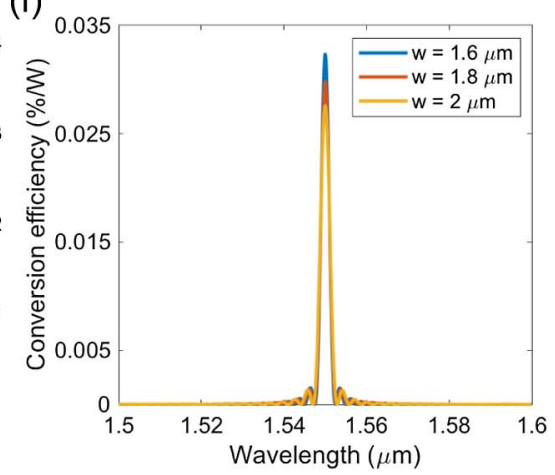

Fig. 1. (a) and (d) Propagation constant mismatch $\Delta \beta$. (b) and (e) CE dependence on wavelength and waveguide width in $0.75 \mu \mathrm{m}$ thick $\mathrm{Si}_{3} \mathrm{~N}_{4}$ waveguide all-optically poled at $1.55 \mu \mathrm{m}$. (c) and (f) $\mathrm{CE}$ as a function of wavelength along the dashed lines in (b) and (e), respectively. Top and bottom rows consider TE- and TM-polarized light, respectively. Grating length $L_{g}$ is $10 \mathrm{~mm}$ and $\chi_{\text {eff }}^{(2)}$ is $0.1 \mathrm{pm} / \mathrm{V}$.
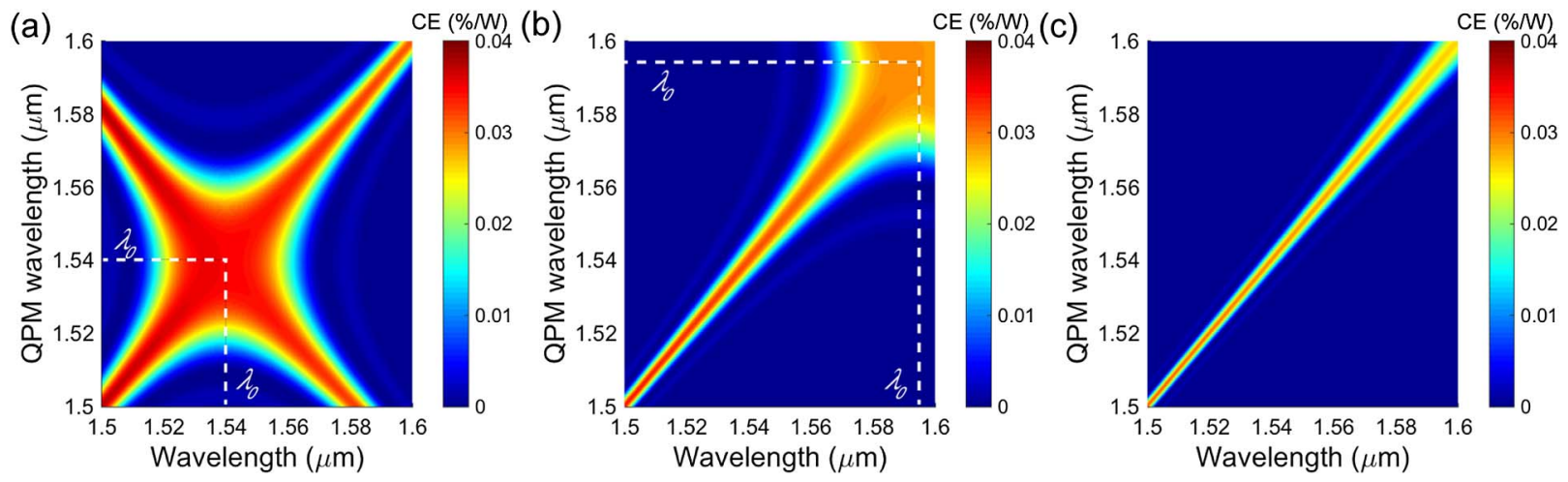

Fig. 2. CE dependence on wavelength in waveguides having different QPM wavelengths. The waveguide height is $0.75 \mu \mathrm{m}$ and widths are (a) $1.6 \mu \mathrm{m}$, (b) $1.8 \mu \mathrm{m}$, and (c) $2.0 \mu \mathrm{m}$. The plots consider TE polarized light; the grating length $L_{g}$ is $10 \mathrm{~mm}$ and $\chi_{\text {eff }}^{(2)}$ is $0.1 \mathrm{pm} / \mathrm{V}$. $\lambda_{0}$ indicates the wavelength at which condition by Eq. (3) is satisfied.

having different QPM conditions and for three cross-sections, namely, $1.6 \mu \mathrm{m} \times 0.75 \mu \mathrm{m}, 1.8 \mu \mathrm{m} \times 0.75 \mu \mathrm{m}$, and $2.0 \mu \mathrm{m} \times$ $0.75 \mu \mathrm{m}$. The plots consider TE polarized light. For the case of the $1.6 \mu \mathrm{m} \times 0.75 \mu \mathrm{m}$ cross-section, $\lambda_{0}$ is estimated near $1.54 \mu \mathrm{m}$ using Eq. (3). The same $\lambda_{0}$ value is obtained from Fig. 2(a). Increasing or reducing the QPM wavelength around $\lambda_{0}$ will cause two peaks to appear in the CE spectrum, which subsequently narrow as this wavelength difference increases. This is also the cause of two local maxima to appear in a plot of CE as a function of wavelength in Fig. 1(c) for QPM in the waveguide with a cross-section of $1.6 \mu \mathrm{m} \times 0.75 \mu \mathrm{m}$. Consequently, widely dissimilar CE spectra can be obtained from a single waveguide by simply re-poling it at a different wavelength. When the QPM wavelength is far from $\lambda_{0}$, as for Figs. 2(b) and 2(c) with $\lambda_{0}$ being at $1.595 \mu \mathrm{m}$ and $1.646 \mu \mathrm{m}$, respectively, the CE bandwidth remains narrow. In this case, re-poling the waveguide at different wavelengths would result in a shift in the QPM spectrum without significantly changing its shape. 
It is important to emphasize that CE estimations, as in Figs. 1 and 2, are extremely sensitive to the refractive index at pump and $\mathrm{SH}$ wavelengths. This implies that the material refractive index dependence on wavelength should be determined experimentally with high precision in order to obtain meaningful results through numerical simulations.

\section{B. Experimental Confirmation}

We investigated experimentally such QPM spectral bandwidth dependence on the waveguide geometry. For this purpose, buried $\mathrm{Si}_{3} \mathrm{~N}_{4}$ waveguides with a height of $0.75 \mu \mathrm{m}$ and widths of $1.6,1.8$, and $2.0 \mu \mathrm{m}$ were fabricated according to the photonic Damascene process [26,33]. The $55 \mathrm{~mm}$ long waveguides are folded in 11 meanders on a $5 \mathrm{~mm} \times 5 \mathrm{~mm}$ chip by using curved sections. The radii of the bends for each waveguide were designed to reduce the influence of mode mixing and had a minimum bend radius of $100 \mu \mathrm{m}$. The waveguides have optical propagation loss of ca. $4 \mathrm{~dB} / \mathrm{m}$ and were all optically poled at $1.55 \mu \mathrm{m}$ as described elsewhere [20]. We use a PID controller and a Peltier module to maintain the temperature of the chip at $30^{\circ} \mathrm{C}$ during the poling procedure. During all-optical poling, an effective $\chi^{(2)}$ grating is inscribed inside the waveguide ensuring QPM as confirmed previously [13]. In Fig. 3, the measured (dotted) and fitted (dashed-dotted lines) CE dependences on wavelength after poling are shown. The effective nonlinearity $\chi_{\text {eff }}^{(2)}$, grating length $L_{g}$, and period $\Lambda$ were found using a least-squares fit of experimental data by Eq. (1), resulting in relative standard error below $2.2 \%$. Here, the numerically simulated propagation constants at pump and $\mathrm{SH}$ wavelengths were provided as functions of wavelength [see Figs. 1(a) and 1(d)] and were kept constant. During poling, we monitored the process such that the $\mathrm{CE}$ at the poling wavelength would be of the same order of magnitude in all waveguides and for all polarizations. From Eq. (1), this means that we expect the grating length $L_{g}$ and strength $\chi_{\text {eff }}^{(2)}$ to be approximately equal in all waveguides. Any change in CE spectral bandwidth could therefore be attributed solely to the difference in dispersion.

The experimentally measured QPM wavelength is slightly shifted relative to the $1.55 \mu \mathrm{m}$ poling wavelength. This is explained by temperature differences in the waveguide during poling performed with a pulsed pump and QPM measurement done with CW light. Despite this slight shift, the QPM spectral responses in Fig. 3 follow the expectations from numerical simulations. Here, the broadest QPM response is obtained in a waveguide with a cross-section of $1.6 \mu \mathrm{m} \times 0.75 \mu \mathrm{m}$ poled using TE-polarized light [see Fig. 3(a)]. The parameters obtained from the fit are included as insets in Fig. 3. The effective nonlinearity $\chi_{\text {eff }}^{(2)}$ and grating length $L_{g}$ are similar for all waveguides. There is a slight difference in Fig. 3(a), where the grating is estimated to be three times longer than in other waveguides. This could be due to two factors. First, we do not have the full CE spectrum since the data points below $1.535 \mu \mathrm{m}$ could not be captured due to limitations of the C-band amplifier (Keopsys CEFA-C-PB-HP, output power $37 \mathrm{dBm}$ ). Second, errors could arise due to the imprecision of the numerically simulated $\Delta \beta$. This is particularly impactful since we expect $\Delta \beta$ to be close to zero in a spectrally broad band around the QPM wavelength, and any small error in $\Delta \beta$ could result in increased uncertainty of $L_{g}$.
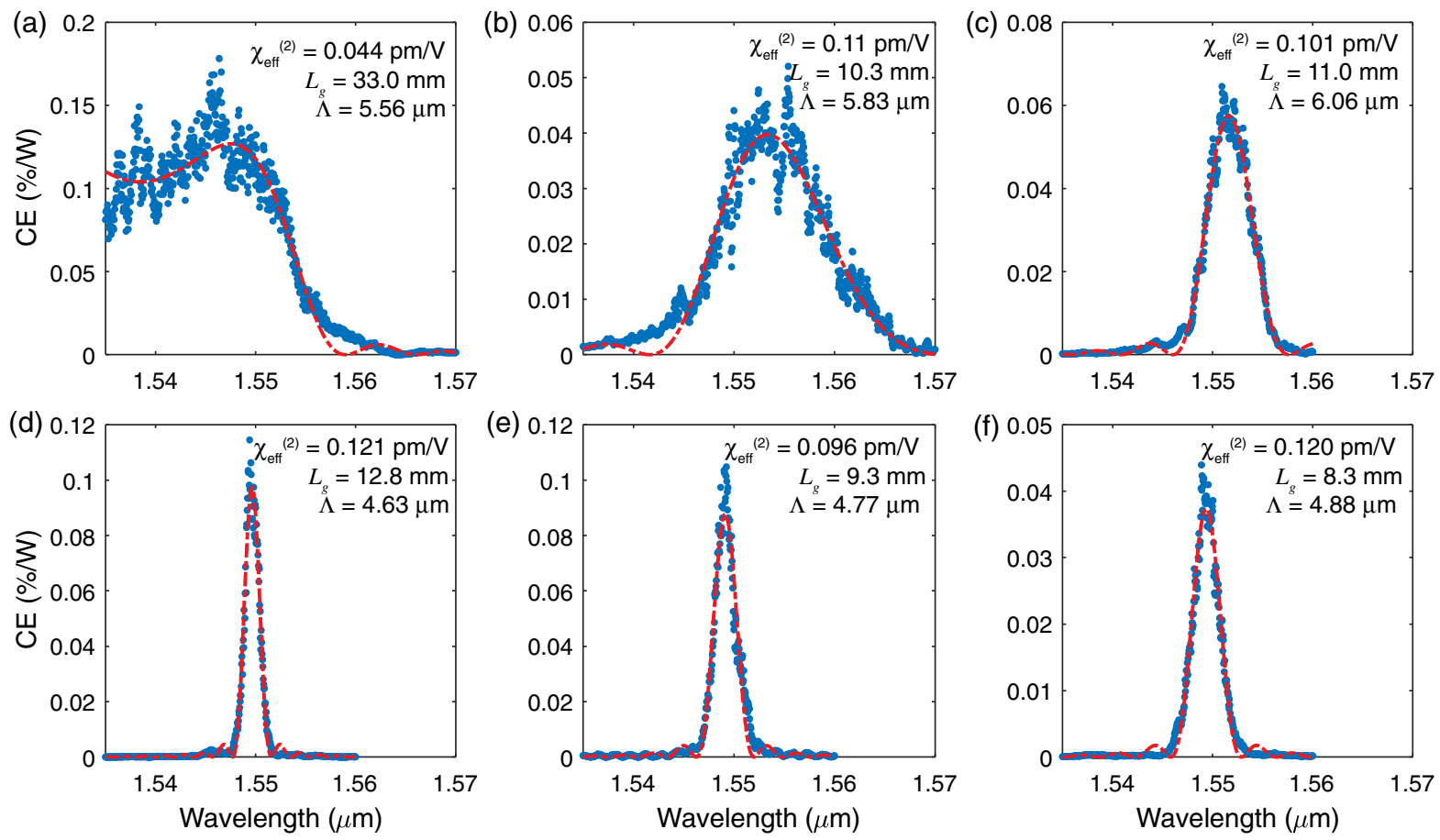

Fig. 3. Experimentally measured CE spectra (blue dots) with fits (red dashed line) of waveguides all-optically poled at $1.55 \mu \mathrm{m}$ with the effective nonlinearity $\chi_{\text {eff }}^{(2)}$, grating length $L_{g}$, and period $\Lambda$ obtained from fit. Waveguide cross-sections are (a) and (d) $1.6 \mu \mathrm{m} \times 0.75 \mu \mathrm{m}$, (b) and (e) $1.8 \mu \mathrm{m} \times 0.75 \mu \mathrm{m}$, and (c) and (f) $2.0 \mu \mathrm{m} \times 0.75 \mu \mathrm{m}$. The polarization is TE for (a)-(c) and TM for (d) -(f) where both polarizations in the waveguides with the same dimensions result in independent parameter sets. 
In the case of TE polarization, the QPM spectrum becomes narrower with the increase of waveguide width from 1.6 to $2.0 \mu \mathrm{m}$ [Figs. 3(a)-3(c)]. The opposite occurs when the same waveguides are poled using a TM-polarized pump [Figs. 3(d)-3(f)]. These experimental results perfectly confirm that dispersion engineering of QPM can be employed to enable both efficient narrowband and broadband responses in the same waveguide by leveraging the two modes of polarization. Most importantly, reaching broadband response does not come at the price of efficiency since long gratings can still be used.

\section{GV MATCHING AND $\boldsymbol{f}_{\text {CEO }}$ RETRIEVAL}

As shown previously, the largest bandwidth of QPM is achieved when Eq. (3) is satisfied. Meeting the requirement by Eq. (3) also ensures GV matching taking place in the waveguide, which, together with QPM, is key requirements for efficient $\mathrm{SH}$ generation of pulsed light [34]. The presence of GV matching was confirmed by calculation of the group index $n_{g \omega}$ dependence on wavelength (see Appendix A). We demonstrate the benefit of simultaneous phase and GV matching by $f_{\mathrm{CEO}}$ detection proof-of-principle experiment. For this purpose, an $f-2 f$ interferometer (for details, see Appendix B) was implemented $[35,36]$. Here, a beam from a femtosecond pulse source is split and delivered to two $\mathrm{Si}_{3} \mathrm{~N}_{4}$ waveguides. One of the waveguides, having dimensions of $2.65 \mu \mathrm{m} \times 0.78 \mu \mathrm{m}$, is dispersion-engineered to generate a TE dispersive wave (DW) at around $0.78 \mu \mathrm{m}$ wavelength where the $\mathrm{SH}$ of the pump is located (for details on DW engineering, see Appendix C). The other beam is delivered to a waveguide with a cross-section of $1.6 \mu \mathrm{m} \times 0.75 \mu \mathrm{m}$, which has a broad CE spectral response after all-optical poling, as shown previously. The spectra of obtained SC and SH from each chip are shown in Fig. 4(a). Both beams leaving the chips are collected and delivered to a high-speed detector that is connected to a frequency analyzer. The retrieved beat notes are displayed in Fig. 4(b). Here, the $f_{\text {rep }}$ corresponds to the repetition rate of the laser at $100 \mathrm{MHz}$. The $f_{\text {CEO }}$ is detected with a signal-to-noise ratio $(\mathrm{SNR})$ at $32.7 \mathrm{~dB}$ with the resolution bandwidth being $50 \mathrm{kHz}$ when the average output powers of the pump and $\mathrm{SH}$ at the output waveguide are $4.2 \mathrm{dBm}$ and $-25.1 \mathrm{dBm}$, respectively. Moreover, the SNR would not reduce during several days of operation, suggesting that such power coupled inside the waveguide would not erase the inscribed nonlinear grating. The obtained SNR is sufficient for ensuring frequency comb stabilization without phase slips; yet, it can be further enlarged by increasing the power coupled to the waveguide in which SH generation is taking place. However, there is an optimal coupled power before SC generation takes place in the poled waveguide that results in the erasure of the grating [13].

\section{DEPENDENCE OF DISPERSION ENGINEERED QPM ON TEMPERATURE}

QPM wavelength variation is enabled by the thermo-optic effect, as shown previously in $\mathrm{LN}$ [37] and $\mathrm{Si}_{3} \mathrm{~N}_{4}$ [20] integrated photonics platforms. The change of the waveguide temperature causes alteration of phase-matching condition and, consequently, the shift of QPM wavelength. The QPM wavelength variation magnitude and sign will also depend on the waveguide geometry and can be estimated from the derivative of $\Delta \beta$ with respect to the pump wavelength $\lambda, \partial(\Delta \beta) / \partial \lambda$. In the vicinity of the poling wavelength, if $\partial(\Delta \beta) / \partial \lambda>0$, then the QPM wavelength will blueshift with the increase of temperature and vice versa. Also, the shift in QPM wavelength will be greater for smaller absolute $\partial(\Delta \beta) / \partial \lambda$. The change in QPM wavelength $\Delta \lambda_{\mathrm{QPM}}$ with small variation of temperature can be approximated as (for illustrative explanation, see Appendix D)

$$
\Delta \lambda_{\mathrm{QPM}}=\left.\frac{\partial(\Delta \beta)}{\partial T}\right|_{\lambda_{\mathrm{QPM}}} \cdot \Delta T /\left[-\left.\frac{\partial(\Delta \beta)}{\partial \lambda}\right|_{\lambda_{\mathrm{QPM}}}\right] .
$$

In Fig. 5(a), we plot the $\partial(\Delta \beta) / \partial \lambda$ dependence on waveguide width in a $0.75 \mu \mathrm{m}$ thick $\mathrm{Si}_{3} \mathrm{~N}_{4}$ waveguide all-optically poled at a QPM wavelength of $1.55 \mu \mathrm{m}$ at a temperature of $30^{\circ} \mathrm{C}$. Data in Fig. 5(a) can also be used to estimate the geometrical parameters of the waveguide core at which the $\mathrm{CE}$ spectral response would be the broadest, meaning whenever (a)

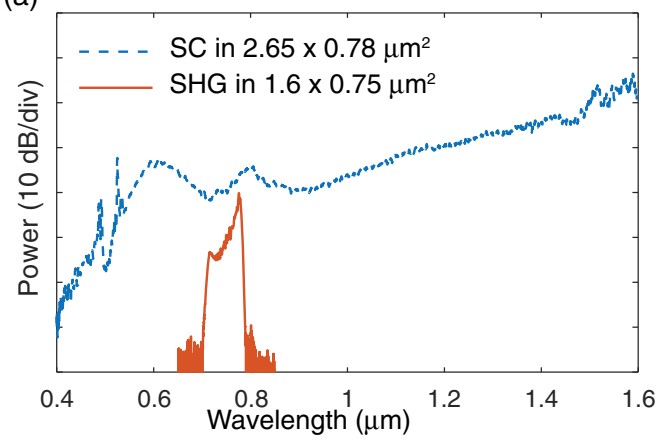

(b)

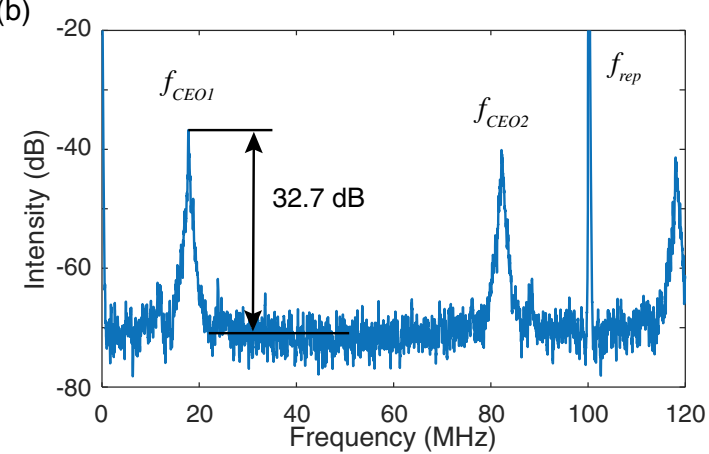

Fig. 4. Carrier-envelope-offset frequency measurement employing an all-optically poled $\mathrm{Si}_{3} \mathrm{~N}_{4}$ waveguide. (a) Spectra of SC generated in a $2.65 \mu \mathrm{m} \times 0.78 \mu \mathrm{m}$ dispersion-engineered waveguide and $\mathrm{SH}$ of femtosecond pulses in all-optically poled $1.6 \mu \mathrm{m} \times 0.75 \mu \mathrm{m}$ waveguide measured by an optical spectrum analyzer (Yokogawa AQ6374). (b) Measured beat notes between SC and SH. The resolution bandwidth of frequency analyzer (Rohde \& Schwarz FSUP Signal Source Analyzer) is $50 \mathrm{kHz}$. 
(a)

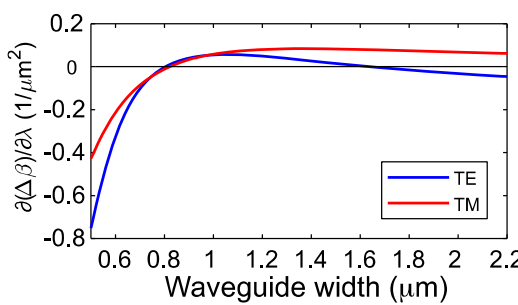

(d)

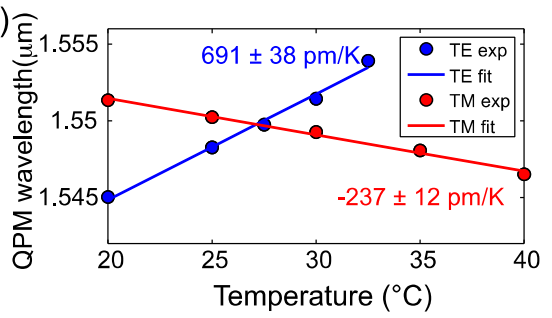

(b)

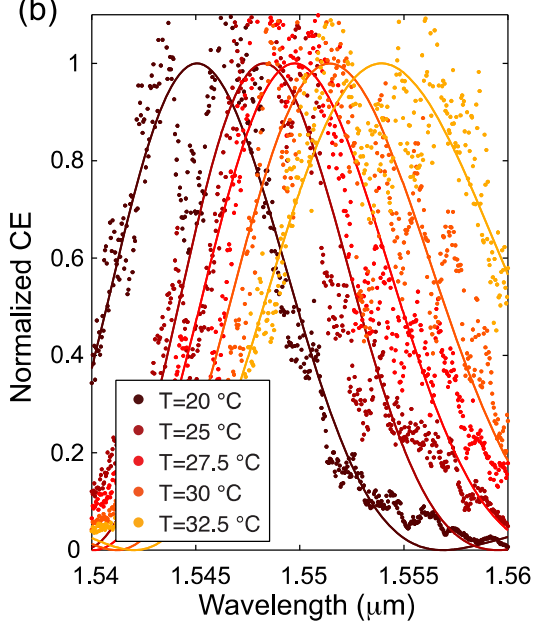

(c)

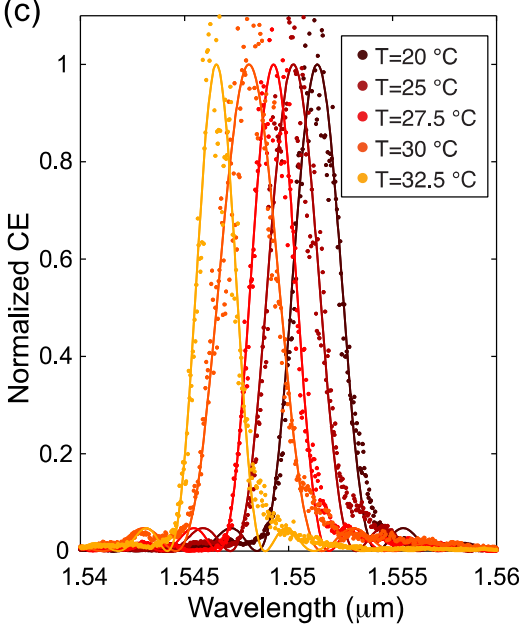

Fig. 5. (a) $\partial(\Delta \beta) / \partial \lambda$ as a function of waveguide width in a $0.75 \mu \mathrm{m}$ thick $\mathrm{Si}_{3} \mathrm{~N}_{4}$ waveguide all-optically poled at $1.55 \mu \mathrm{m}$. When the $\partial(\Delta \beta) / \partial \lambda$ is positive in the waveguide around poling wavelength, the QPM wavelength will blueshift with the increase of temperature, and vice versa. (b) and (c) Measurements (points) and fits (lines) of CE spectra at different temperatures in poled $1.8 \mu \mathrm{m} \times 0.75 \mu \mathrm{m}$ waveguide for operation using TE or TM polarized light, respectively. (d) Extracted QPM wavelength as well as temperature dependence and linear fits. Tuning slopes $\Delta \lambda / \Delta T$ calculated from linear fits are $691 \pm 38 \mathrm{pm} / \mathrm{K}$ and $-237 \pm 12 \mathrm{pm} / \mathrm{K}$ for TE and TM polarized light, respectively.

$\partial(\Delta \beta) / \partial \lambda$ is near 0 . For example, for the case of a $0.75 \mu \mathrm{m}$ thick $\mathrm{Si}_{3} \mathrm{~N}_{4}$ waveguide, the broadest CE for TE polarized light will be present in waveguides with widths of 0.8 and $1.595 \mu \mathrm{m}$; for TM, the waveguide width will be around $0.82 \mu \mathrm{m}$.

From Fig. 5(a) and Eq. (4), we can expect that, in a $1.8 \mu \mathrm{m} \times 0.75 \mu \mathrm{m}$ waveguide, the QPM wavelength should redshift (blueshift) with the increase of temperature if TE (TM) polarized light is used. In order to confirm this, we alloptically poled a waveguide with a cross-section of $1.8 \mu \mathrm{m} \times$ $0.75 \mu \mathrm{m}$ using $1.55 \mu \mathrm{m}$ light polarized at TE and TM and measured the QPM spectrum with a CW wave for different temperature settings. The $\mathrm{SH}$ spectra at several temperatures in the range from $20^{\circ} \mathrm{C}$ to $40^{\circ} \mathrm{C}$ for TE and TM polarized light are plotted in Figs. 5(b) and 5(c), respectively. The CE spectra were fitted according to Eq. (1) and normalized to maximal value of the fit function. From location of CE maxima at different chip temperatures, the QPM wavelengths are extracted and plotted in Fig. 5(d). We experimentally confirm that, in case QPM is induced for TE polarized light, the QPM wavelength is redshifted with the increase of temperature. The slope of this change is $691 \pm 38 \mathrm{pm} / \mathrm{K}$. When the waveguide is all-optically poled using TM polarized light, the QPM wavelength is blueshifted with increase of temperature and changes with $-237 \pm 12 \mathrm{pm} / \mathrm{K}$.

\section{CONCLUSIONS}

Implementing QPM in waveguiding structures allows for extra degrees of freedom. Here, the possibility to vary the waveguide geometry is leveraged to adjust and control the QPM properties in $\mathrm{Si}_{3} \mathrm{~N}_{4}$ waveguides. Experimentally, the QPM inside the waveguides is achieved using an all-optical poling technique that is simple to implement and does not require traditionally applied complex nanostructuring techniques.
Through optimization of waveguide core dimensions, we modify the dispersion of the waveguides to achieve broadband and efficient frequency conversion in waveguides with QPM. The CE bandwidth can be increased by minimizing gradients of propagation constant mismatch $\Delta \beta$ with respect to the wavelength. Meeting the mentioned condition in a waveguide with QPM results not only in broadband phase-matching but also in GV matching. The latter provides the possibility of processing large bandwidth optical signals such as ultrashort pulses. We employ this phenomenon to retrieve the $f_{\text {CEO }}$ using an $f-2 f$ interferometer technique as a proofof-principle experiment. The obtained SNR, the measurement with resolution bandwidth being $50 \mathrm{kHz}$ is $32.7 \mathrm{~dB}$, sufficient for frequency comb phase stabilization. Finally, we show that the waveguide dispersion determines the QPM wavelength variation magnitude and sign with temperature. The absolute QPM wavelength variation with temperature is larger for small $|\partial(\Delta \beta) / \partial \lambda|$, while the sign of QPM wavelength change with temperature is determined by that of $\partial(\Delta \beta) / \partial \lambda$ near the QPM wavelength. In case the slope is positive, the QPM wavelength will blueshift with increase of the temperature and vice versa. This is experimentally confirmed by measuring the QPM wavelength variation due to thermo-optic effect in a waveguide with a cross-section of $1.8 \mu \mathrm{m} \times 0.75 \mu \mathrm{m}$ at TE and TM polarizations, respectively. In this case, the $\Delta \beta$ gradients with respect to wavelength vary both in sign and magnitude for each polarization. For poling with TE polarized light, the QPM wavelength redshifts with the increase of temperature with a slope of $691 \mathrm{pm} / \mathrm{K}$. For poling with TM polarized light, the QPM wavelength blueshifts with $-237 \mathrm{pm} / \mathrm{K}$. While the experimental validations were carried out in all optically poled $\mathrm{Si}_{3} \mathrm{~N}_{4}$ waveguides, it is important to note that the obtained rules and properties are general and can be applied for any waveguide platform and any poling mechanism. 


\section{APPENDIX A: GROUP INDEX CALCULATIONS}

The group index for TE-polarized light was numerically calculated according to the following equation

$$
n_{g \omega}=n_{\omega}-\frac{\mathrm{d} n_{\omega}}{\mathrm{d} \lambda} \lambda .
$$

In Eq. (A1), $n_{\omega}$ was calculated by a numerical mode solver. In Fig. 6(a), we plot $n_{g \omega}$ as a function of wavelength calculated at different $\mathrm{Si}_{3} \mathrm{~N}_{4}$ waveguide widths and fixed waveguide height of $0.75 \mu \mathrm{m}$. Due to availability of a femtosecond pulsed source operating at $1.56 \mu \mathrm{m}$, which we were using in measurements, we calculated the difference between group indexes $\Delta n_{g}$ at 0.78 and $1.56 \mu \mathrm{m}$ as a function of the $\mathrm{Si}_{3} \mathrm{~N}_{4}$ waveguide width [Fig. 6(b)]. As evident, the group index difference at these wavelengths is expected to be near zero in a waveguide with a cross-section of $1.6 \mu \mathrm{m} \times 0.75 \mu \mathrm{m}$, while perfect GV matching would be achieved in a waveguide with core dimensions $1.546 \mu \mathrm{m} \times 0.75 \mu \mathrm{m}$.

\section{APPENDIX B: $\boldsymbol{f}_{\mathrm{CEO}}$ MEASUREMENT}

The $f_{\mathrm{CEO}}$ measurement was carried out using the setup in Fig. 7. The femtosecond pulses from PM fiber are collimated and then split using a beam splitter. The polarization is controlled with a half-wave plate. Using optical lenses, we couple both beams into two waveguides. One of the waveguides has a cross-section of $1.6 \mu \mathrm{m} \times 0.75 \mu \mathrm{m}$ and after poling has a broadband CE response at $1.55 \mu \mathrm{m}$ wavelength. At the output of the chip, we measure SH spectra, as shown in Fig. 4(a). The other chip features a dispersion-engineered $5 \mathrm{~mm}$ long $\mathrm{Si}_{3} \mathrm{~N}_{4}$ waveguide used to generate broad SC, including DW centered at $0.78 \mu \mathrm{m}$ wavelength (see Appendix $\mathrm{C}$ for details). The output light from the $\mathrm{Si}_{3} \mathrm{~N}_{4}$ waveguides is delivered to another beam splitter and aligned to be overlapping and collinear in space so that beams can interfere. A delay line in the path of $\mathrm{SH}$ is used to control the walk-off between pulses. To retrieve the $f_{\mathrm{CEO}}$ beat note, the walk-off should be close to zero. The interfering beams are collected and delivered to a detecting

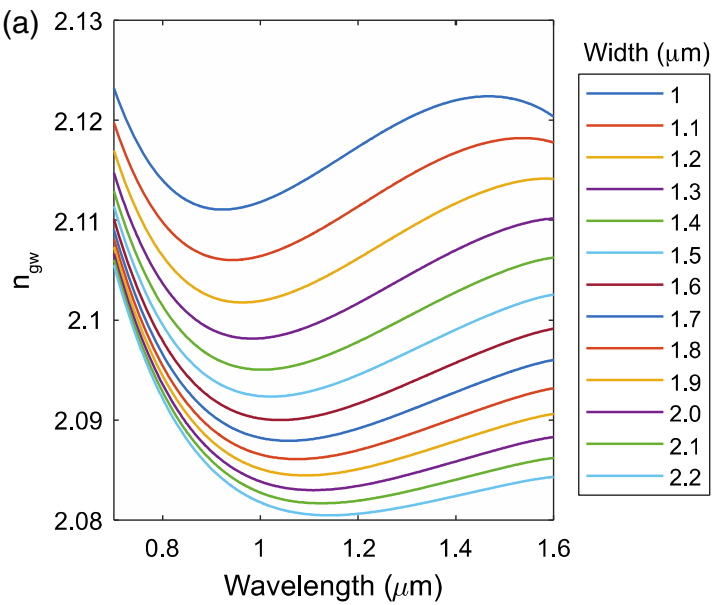

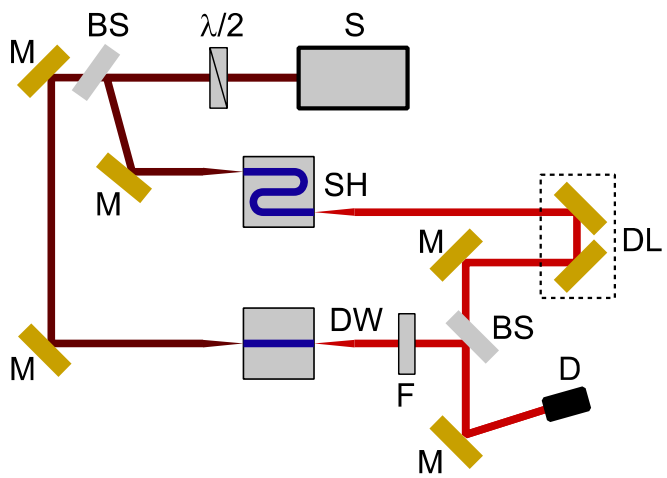

Fig. 7. Setup for femtosecond laser $f_{\mathrm{CEO}}$ retrieval. $S$ is the femtosecond light source (FemtoFErb 1560 from Toptica); $\lambda / 2$, half-wave plate for polarization control; BS, beam splitter; $\mathrm{M}$, gold coated mirror; $\mathrm{F}$, a set of dichroic filters transmitting between 0.6 and $0.95 \mu \mathrm{m}$; $\mathrm{DL}$, delay line; $\mathrm{D}$, detecting unit, including fiber coupler, single-mode fiber, high-speed detector (FPD610-FC-VIS from Menlo Systems), and frequency analyzer. SH and DW stand for second harmonic and dispersive wave, respectively.

unit, which consists of a collimator, a single-mode fiber, and a high-speed detector connected to a frequency analyzer.

\section{APPENDIX C: DISPERSION ENGINEERING FOR OPTICAL-FREQUENCY-COMB SC GENERATION}

The waveguide used for $\mathrm{SC}$ generation in the $f_{\mathrm{CEO}}$ retrieval experiment was designed to support soliton formation as well as dispersive wave generation when pumped with a femtosecond light source at $1.56 \mu \mathrm{m}$. The employed design rules and methods follow those described elsewhere [22]. The waveguide is made such that it has anomalous dispersion for a TEpolarized pump. Using a numerical mode solver, we calculate the integrated dispersion $\beta_{\text {int }}$ as a function of wavelength and tailor it by changing the waveguide geometry to be at the $\mathrm{SH}$ wavelength of the pump. The integrated dispersion displays the phase mismatch between a soliton, which is formed within the

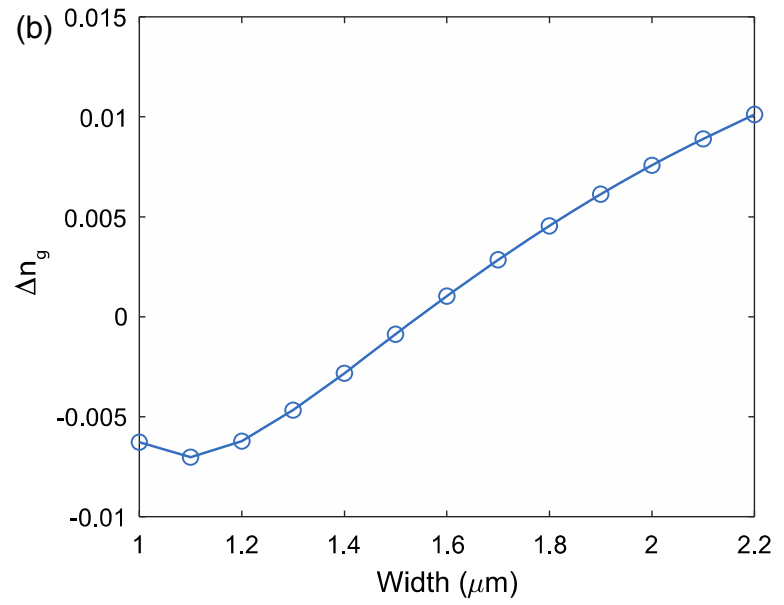

Fig. 6. (a) Group index $n_{g \omega}$ of TE-polarized light as a function of wavelength calculated at different $\mathrm{Si}_{3} \mathrm{~N}_{4}$ waveguide widths and fixed waveguide height of $0.75 \mu \mathrm{m}$. (b) Difference between group indexes $\Delta n_{g}$ at 0.78 and $1.56 \mu \mathrm{m}$ as a function of $\mathrm{Si}_{3} \mathrm{~N}_{4}$ waveguide width having height of $0.75 \mu \mathrm{m}$. 
(a)

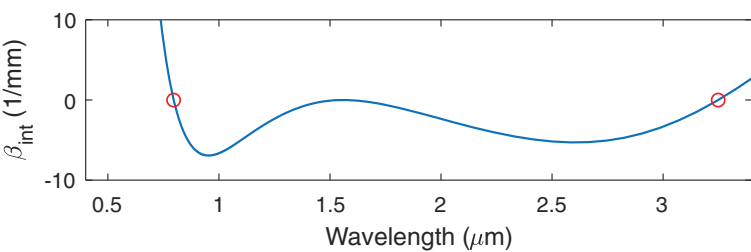

(b)

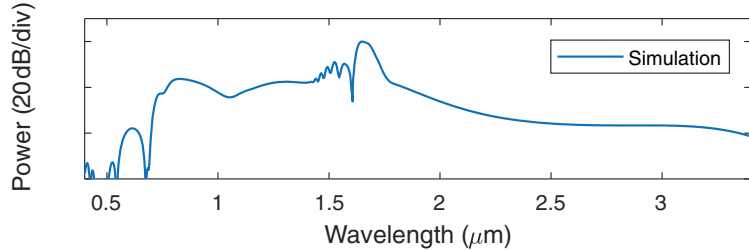

(c)

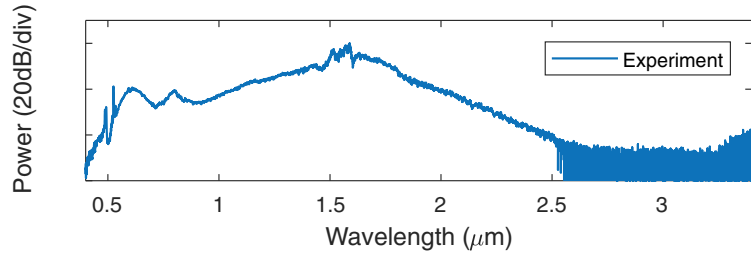

Fig. 8. (a) Calculated integrated dispersion $\beta_{\text {int }}$ for waveguide with cross-section $2.65 \mu \mathrm{m} \times 0.78 \mu \mathrm{m} \mathrm{Si}_{3} \mathrm{~N}_{4}$ waveguide. (b) Numerically calculated SC generated in a waveguide with cross-section $2.65 \mu \mathrm{m} \times$ $0.78 \mu \mathrm{m}$ and (c) measured SC spectrum at the output of a $5 \mathrm{~mm}$ long straight waveguide with $15 \mathrm{dBm}$ average power in the waveguide.

waveguide, and DW. When the integrated dispersion is equal to zero, the soliton and DW are phase-matched and efficient energy transfer from soliton to DW can occur. In Fig. 8(a), we show the integrated dispersion as a function of wavelength for a waveguide with a cross-section of $2.65 \mu \mathrm{m} \times 0.78 \mu \mathrm{m}$, where the $\beta_{\text {int }}$ curve crosses zero at $0.78 \mu \mathrm{m}$. The generated SC spectrum in such a $5 \mathrm{~mm}$ long waveguide was calculated using a nonlinear Schrödinger equation and is as shown in Fig. 8(b). The experimentally measured SC spectrum [see Fig. 8(c)] in such a waveguide is in good general agreement with the numerical simulations.

\section{APPENDIX D: THERMAL TUNING AND QPM WAVELENGTH SHIFT}

An illustrative explanation of the variation of QPM wavelength with temperature is shown in Fig. 9. We consider the case where the propagation constant mismatch $\Delta \beta$ decreases with wavelength. Here, the increase of temperature will cause the $\Delta \beta$ curve to shift upward, resulting in QPM wavelength to redshift. It is important to note that we have applied the following considerations in the description above. First, we consider the thermal expansion contribution, which would result in the grating period change, to be relatively small in comparison with the thermo-optic effect and therefore can be ignored [38]. Second, we assume that the thermo-optic coefficient $\partial\left(n_{\text {eff }}\right) / \partial T$ at $\mathrm{SH}$ is greater than that at pump wavelength or, in other words, that $\partial(\Delta \beta) / \partial T>0$. The latter was experimentally confirmed to be true as shown in Fig. 5(d) of the main text.

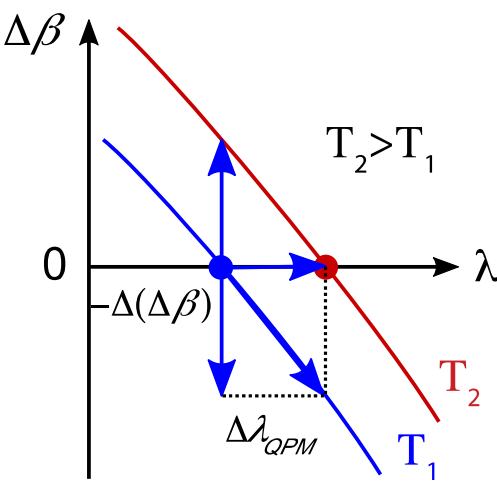

Fig. 9. Propagation constant mismatch $\Delta \beta$ as a function of wavelength. If $\Delta \beta$ decreases with wavelength, then temperature change from $T_{1}$ to $T_{2}$, where $T_{2}>T_{1}$, will cause the QPM wavelength to redshift or $\Delta \lambda_{\mathrm{QPM}}>0$. The opposite happens if $\Delta \beta$ increases with wavelength.

Funding. Schweizerischer Nationalfonds zur Förderung der Wissenschaftlichen Forschung (176563) (BRIDGE); U.S. Air Force (FA9550-19-1-0250); Defense Advanced Research Projects Agency (HR0011-15-C-055) (DODOS); European Research Council (ERC-2017-CoG 771647) (PISSARRO); Microsystems Technology Office (MTO); Air Force Material Command; Air Force Office of Scientific Research; EPFL Center of MicroNanoTechnology (CMi).

Acknowledgment. We acknowledge J. He and T. Liu for assistance in the sample test.

Disclosures. The authors declare no conflicts of interest.

\section{REFERENCES}

1. J. Leuthold, C. Koos, and W. Freude, "Nonlinear silicon photonics," Nat. Photonics 4, 535-544 (2010).

2. E. Timurdogan, C. V. Poulton, M. J. Byrd, and M. R. Watts, "Electric field-induced second-order nonlinear optical effects in silicon waveguides," Nat. Photonics 11, 200-206 (2017).

3. A. L. Gaeta, M. Lipson, and T. J. Kippenberg, "Photonic-chip-based frequency combs," Nat. Photonics 13, 158-169 (2019).

4. D. J. Moss, R. Morandotti, A. L. Gaeta, and M. Lipson, "New CMOScompatible platforms based on silicon nitride and Hydex for nonlinear optics," Nat. Photonics 7, 597-607 (2013).

5. C. Wang, C. Langrock, A. Marandi, M. Jankowski, M. Zhang, B. Desiatov, M. M. Fejer, and M. Lončar, "Ultrahigh-efficiency wavelength conversion in nanophotonic periodically poled lithium niobate waveguides," Optica 5, 1438-1441 (2018).

6. C. Wang, M. Zhang, X. Chen, M. Bertrand, A. Shams-Ansari, S. Chandrasekhar, P. Winzer, and M. Lončar, "Integrated lithium niobate electro-optic modulators operating at CMOS-compatible voltages," Nature 562, 101-104 (2018).

7. C. Xiong, W. H. P. Pernice, and H. X. Tang, "Low-loss, silicon integrated, aluminum nitride photonic circuits and their use for electrooptic signal processing," Nano Lett. 12, 3562-3568 (2012).

8. B. Dong, X. Luo, S. Zhu, M. Li, D. Hasan, L. Zhang, S. J. Chua, J. Wei, Y. Chang, G.-Q. Lo, K. W. Ang, D.-L. Kwong, and C. Lee, "Aluminum nitride on insulator (AINOI) platform for mid-infrared photonics," Opt. Lett. 44, 73-76 (2019).

9. A. W. Bruch, X. Liu, X. Guo, J. B. Surya, Z. Gong, L. Zhang, J. Wang, J. Yan, and H. X. Tang, "17000\%/W second-harmonic conversion 
efficiency in single-crystalline aluminum nitride microresonators," Appl. Phys. Lett. 113, 131102 (2018).

10. J. Lu, J. B. Surya, X. Liu, A. W. Bruch, Z. Gong, Y. Xu, and H. X. Tang, "Periodically poled thin-film lithium niobate microring resonators with a second-harmonic generation efficiency of $250,000 \% / \mathrm{W}$," Optica 6 , 1455-1460 (2019).

11. M. M. Fejer, G. A. Magel, D. H. Jundt, and R. L. Byer, "Quasi-phasematched second harmonic generation: tuning and tolerances," IEEE J. Quantum Electron. 28, 2631-2654 (1992).

12. A. Billat, D. Grassani, M. H. P. Pfeiffer, S. Kharitonov, T. J. Kippenberg, and C. S. Brès, "Large second harmonic generation enhancement in $\mathrm{Si}_{3} \mathrm{~N}_{4}$ waveguides by all-optically induced quasi-phasematching," Nat. Commun. 8, 1016 (2017).

13. E. Nitiss, T. Liu, D. Grassani, M. Pfeiffer, T. J. Kippenberg, and C.-S. Brès, "Formation rules and dynamics of photo-induced $\chi^{(2)}$ gratings in silicon nitride waveguides," ACS Photon. 7, 147-153 (2019).

14. M. A. G. Porcel, J. Mak, C. Taballione, V. K. Schermerhorn, J. P. Epping, P. J. M. van der Slot, and K.-J. Boller, "Photo-induced second-order nonlinearity in stoichiometric silicon nitride waveguides," Opt. Express 25, 33143-33159 (2017).

15. M. A. G. Porcel, J. Mak, C. Taballione, V. K. Schermerhorn, J. P. Epping, P. J. M. van der Slot, and K.-J. Boller, "Photoinduced $\chi^{(2)}$ for second harmonic generation in stoichiometric silicon nitride waveguides," Proc. SPIE 10228, 102280R (2017).

16. D. D. Hickstein, D. R. Carlson, H. Mundoor, J. B. Khurgin, K. Srinivasan, D. Westly, A. Kowligy, I. I. Smalyukh, S. A. Diddams, and S. B. Papp, "Self-organized nonlinear gratings for ultrafast nanophotonics," Nat. Photonics 13, 494-499 (2019).

17. D. Z. Anderson, V. Mizrahi, and J. E. Sipe, "Model for secondharmonic generation in glass optical fibers based on asymmetric photoelectron emission from defect sites," Opt. Lett. 16, 796-798 (1991),

18. N. B. Baranova, B. Y. Zel'dovich, A. N. Chudinov, and A. A. Shul'ginov, "Theory and observation of polar asymmetry of photoionization in a field with $<\mathrm{E}^{3}>\neq 0$," Zh. Eksp. Teor. Fiz. 98, 1857-1868 (1990).

19. E. M. Dianov and D. S. Starodubov, "Photoinduced generation of the second harmonic in centrosymmetric media," Quantum Electron. 25, 395-407 (1995).

20. E. Nitiss, O. Yakar, A. Stroganov, and C.-S. Brès, "Highly tunable second-harmonic generation in all-optically poled silicon nitride waveguides," Opt. Lett. 45, 1958-1961 (2020).

21. A. R. Johnson, A. S. Mayer, A. Klenner, K. Luke, E. S. Lamb, M. R. E. Lamont, C. Joshi, Y. Okawachi, F. W. Wise, M. Lipson, U. Keller, and A. L. Gaeta, "Octave-spanning coherent supercontinuum generation in a silicon nitride waveguide," Opt. Lett. 40, 5117-5120 (2015).

22. H. Guo, C. Herkommer, A. Billat, D. Grassani, C. Zhang, M. H. P. Pfeiffer, W. Weng, C.-S. Brès, and T. J. Kippenberg, "Mid-infrared frequency comb via coherent dispersive wave generation in silicon nitride nanophotonic waveguides," Nat. Photonics 12, 330-335 (2018).

23. D. Grassani, E. Tagkoudi, H. Guo, C. Herkommer, F. Yang, T. J. Kippenberg, and C.-S. Brès, "Mid infrared gas spectroscopy using efficient fiber laser driven photonic chip-based supercontinuum," Nat. Commun. 10, 1553 (2019).

24. J. P. Epping, T. Hellwig, M. Hoekman, R. Mateman, A. Leinse, R. G. Heideman, A. van Rees, P. J. M. van der Slot, C. J. Lee, C. Fallnich, and K.-J. Boller, "On-chip visible-to-infrared supercontinuum generation with more than $495 \mathrm{THz}$ spectral bandwidth," Opt. Express 23, 19596-19604 (2015).

25. T. J. Kippenberg, R. Holzwarth, and S. A. Diddams, "Microresonatorbased optical frequency combs," Science 332, 555-559 (2011).

26. J. Liu, A. S. Raja, M. Karpov, B. Ghadiani, M. H. P. Pfeiffer, B. Du, N. J. Engelsen, H. Guo, M. Zervas, and T. J. Kippenberg, "Ultralow-power chip-based soliton microcombs for photonic integration," Optica 5, 1347-1353 (2018).

27. X. Ji, F. A. S. Barbosa, S. P. Roberts, A. Dutt, J. Cardenas, Y. Okawachi, A. Bryant, A. L. Gaeta, and M. Lipson, "Ultra-low-loss on-chip resonators with sub-milliwatt parametric oscillation threshold," Optica 4, 619-624 (2017).

28. Y. Xuan, Y. Liu, L. T. Varghese, A. J. Metcalf, X. Xue, P.-H. Wang, K. Han, J. A. Jaramillo-Villegas, A. Al Noman, C. Wang, S. Kim, M. Teng, Y. J. Lee, B. Niu, L. Fan, J. Wang, D. E. Leaird, A. M. Weiner, and M. Qi, "High-Q silicon nitride microresonators exhibiting low-power frequency comb initiation," Optica 3, 1171-1180 (2016).

29. Z. Ye, K. Twayana, P. A. Andrekson, and V. Torres-Company, "High$Q \mathrm{Si}_{3} \mathrm{~N}_{4}$ microresonators based on a subtractive processing for Kerr nonlinear optics," Opt. Express 27, 35719-35727 (2019).

30. S. Ramelow, A. Farsi, S. Clemmen, D. Orquiza, K. Luke, M. Lipson, and A. L. Gaeta, "Silicon-nitride platform for narrowband entangled photon generation," arXiv:1508.04358 (2015).

31. C. Reimer, M. Kues, P. Roztocki, B. Wetzel, F. Grazioso, B. E. Little, S. T. Chu, T. Johnston, Y. Bromberg, L. Caspani, D. J. Moss, and R Morandotti, "Generation of multiphoton entangled quantum states by means of integrated frequency combs," Science 351, 1176-1180 (2016).

32. M. Jankowski, C. Langrock, B. Desiatov, A. Marandi, C. Wang, M. Zhang, C. R. Phillips, M. Lončar, and M. M. Fejer, "Ultrabroadband nonlinear optics in nanophotonic periodically poled lithium niobate waveguides," Optica 7, 40-46 (2020).

33. M. H. P. Pfeiffer, J. Liu, A. S. Raja, T. Morais, B. Ghadiani, and T. J. Kippenberg, "Ultra-smooth silicon nitride waveguides based on the Damascene reflow process: fabrication and loss origins," Optica 5, 884-892 (2018)

34. L. Cai, A. V. Gorbach, Y. Wang, H. Hu, and W. Ding, "Highly efficient broadband second harmonic generation mediated by mode hybridization and nonlinearity patterning in compact fiber-integrated lithium niobate nano-waveguides," Sci. Rep. 8, 12478 (2018).

35. G. Steinmeyer, D. H. Sutter, L. Gallmann, N. Matuschek, U. Keller, J. L. Hall, and S. T. Cundiff, "Frontiers in ultrashort pulse generation: pushing the limits in linear and nonlinear optics," Science 286, 15071512 (1999).

36. H. R. Telle, G. Steinmeyer, A. E. Dunlop, J. Stenger, D. H. Sutter, and U. Keller, "Carrier-envelope offset phase control: a novel concept for absolute optical frequency measurement and ultrashort pulse generation," Appl. Phys. B 69, 327-332 (1999).

37. R. Luo, Y. He, H. Liang, M. Li, and Q. Lin, "Highly tunable efficient second-harmonic generation in a lithium niobate nanophotonic waveguide," Optica 5, 1006-1011 (2018).

38. X. Xue, Y. Xuan, C. Wang, P.-H. Wang, Y. Liu, B. Niu, D. E. Leaird, M. $\mathrm{Qi}$, and A. M. Weiner, "Thermal tuning of Kerr frequency combs in silicon nitride microring resonators," Opt. Express 24, 687-698 (2016). 